\title{
HOLOGRAPHIC RECORDING WITH THE USE OF BISTABLE CENTERS IN $\mathrm{CdF}_{2}$
}

\author{
B. KoziARSkA ${ }^{a}$, J.M. LANGeR ${ }^{a}$, A.I. Ryskin ${ }^{b}$, A.S. ShCheUlin ${ }^{b}$ \\ AND A. SUCHOCKI ${ }^{a}$ \\ ${ }^{a}$ Institute of Physics, Polish Academy of Sciences \\ Al. Lotników 32/46, Warszawa, Poland \\ "S.I. Vavilov" State Optical Institute \\ 12 Birzhevaya Line, 199034 St. Petersburg, Russia
}

We show that bistability and metastability of $\mathrm{In}$ and $\mathrm{Ga}$ impurities in $\mathrm{CdF}_{2}$ crystals can be efficiently used in writing volume holographic gratings. Phototransformation of these impurities from the localized to the hydrogenic state leads to a large change of the local polarizability of the host, and thus a significant change of the refractive index. While CdF2:In can be used only at low temperatures (below $150 \mathrm{~K}$ ), $\mathrm{CdF}_{2}$ : Ga crystals are suitable for room temperature recording.

PACS numbers: $42.40 . \mathrm{Lx}, 42.40 . \mathrm{Ht}, 78.20 . \mathrm{Ci}, 78.50 . \mathrm{Ge}$

In and $\mathrm{Ga}$ are well characterised bistable centers in $\mathrm{CdF}_{2}$ crystals $[1,2]$. Their ground state is a localized one, while all excited states are hydrogenic. Due to a large vibronic barrier separating these states these impurities exhibit metastability: In at temperatures below $100 \mathrm{~K}$, while $\mathrm{Ga}$ below $250 \mathrm{~K}$. One might expect that a phototransformation of the In impurity from the ground, localized state, to a metastable, hydrogenic state should lead to the change of the refractive index, and thus be used in efficient writing of a dispersive grating. Due to a very low mobility of electrons in $\mathrm{CdF}_{2}$ and a localized character of the phototransformation process, the ultimate spatial resolution of writing may be limited only by statistical doping fluctuations.

The photoionization of the two types of states occurs in different spectral regions. At room temperature two strongly asymmetric bands are seen [1]. The absorption band in the visible range is caused by the photoionization of the localized ground state, while the IR band, peaked at $\lambda \approx 8 \mu \mathrm{m}$, is due to photoionization of a hydrogenic, extended state. The oscillator strength for both transitions is close to unity. At low temperatures the IR band disappears, unless the crystal is first illuminated by light resonant with the VIS photoionization band. Such illumination causes metastable bleaching of the VIS absorption and simultaneous appearance of the IR band, with 20-30-fold increase in the IR absorption in comparison to the 
room temperature IR absorption. Metastability is facilitated by a barrier separating the two impurity states. The barrier originates from a large lattice relaxation around the impurity upon ionization [3].

The very strong change in the electron localization leads to a change in the local polarizability, and thus a change in the refractive index of the host. The magnitude of the change can be easily estimated from a simple two oscillator model [4]. The probing beam (He-Ne laser light) falls between the two absorption bands, hence both transitions contribute in phase to the changes of the refractive index. Assuming a unity oscillator strength of both transitions one may expect a change of the refractive index for a red-light probe beam about $10^{-4}$ for the change in the impurity population about $10^{18} \mathrm{~cm}^{-3}$ [4], the condition easily met in semiconducting $\mathrm{CdF}_{2}$ crystals doped with either In or $\mathrm{Ga}$.

$\mathrm{CdF}_{2}$ crystals doped with In were grown by the Bridgman method and annealed in $\mathrm{Cd}$ or $\mathrm{K}$ vapors reduce the concentration of $\mathrm{F}^{-}$acceptors. The released electrons are subsequently trapped by the $\mathrm{In}^{3+}$ donors and the crystal becomes conducting. The concentration of uncompensated (optically active) donors estimated from the absorption spectrum was about $10^{18} \mathrm{~cm}^{-3}$.

The measurement of the refractive index changes was made with the use of a Jamin interferometer. The $\mathrm{CdF}_{2}: \mathrm{In}$ crystal was mounted on a cold finger of a liquid nitrogen cryostat, placed between the mirrors of the interferometer. The crystal was illuminated by a second harmonic $(\lambda=532 \mathrm{~nm})$ of a pulsed Nd:YAG laser. The change of the refractive index was measured at the wavelength of $\lambda=0.635 \mu \mathrm{m}$ of a He-Ne laser. The saturation value of $\Delta n=-1.3 \times 10^{-4}$ agrees well with the estimations presented above.

Having thus established a large effect in $\mathrm{CdF}_{2}$ :In semiconducting crystals we performed a standard holographic experiment of writing a grating by two intersecting beams of a CW Innova Ar-laser $(\lambda=514 \mathrm{~nm})$. The He-Ne laser was used as a probe. The experiment was then repeated with $\mathrm{CdF}_{2}: \mathrm{Ga}$ crystals.

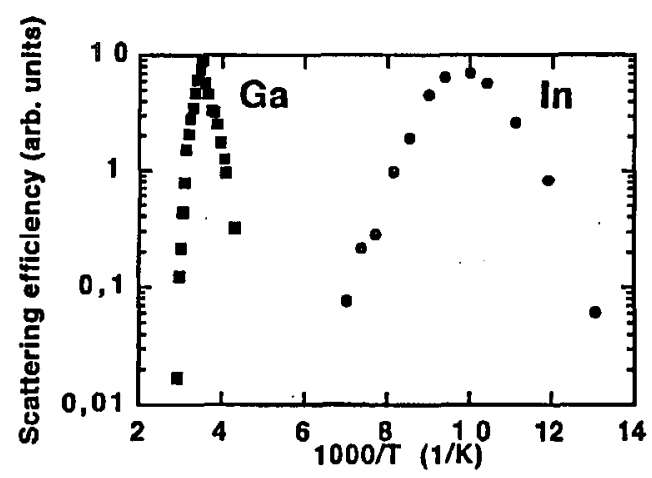

Fig. 1. Temperature dependence of the efficiency of a grating photoionduced in $\mathrm{CdF}_{2}: \mathrm{In}$ or Ga crystals by the low power crossed Ar laser beams $(\lambda=514 \mathrm{~nm})$. 
The lifetime and efficiency of the grating depends critically on temperature. The maximum of the diffraction efficiency occurs at about $120 \mathrm{~K}$ for In and about $280 \mathrm{~K}$ for Ga (Fig. 1). At higher temperatures the decay time and efficiency of the photogenerated grating decreases due to spontaneous thermal recovery of the In impurity from the photoexcited shallow state to the ground state. The activation energy of the high temperature wing equals approximately twice the barrier height (grating efficiency is proportional to the square of the photoinduced change of the refractive index). At lower temperatures a stray VIS light causes bleaching of the VIS absorption band, and erases the photoinduced grating. In our case such a stray light, came mostly from the nonequal intensities of the two writing beams and the laser light scattered from crystal surfaces and cryostat windows. In the case of $\mathrm{Ga}$, doping was less uniform and this added to a smaller diffraction efficiency. However, in both cases the diffraction efficiency at maximum was larger than $10 \%$, thus testifying a phase (refractive index modulation) character of the photoinduced gratings.

A similar effect based on defect metastability was reported recently in AlGaAs epitaxial layers [5]. There, the change of the refractive index was caused by photoionization of DX centers and metastable photogeneration of free carriers. In our case the contribution of free carriers is minimal, if any, and the effect is caused by a phototransformation of the impurity itself. In contrast to AlGaAs layers bulk $\mathrm{CdF}_{2}$ crystals can easily be grown, therefore they are more suitable for writing thick and multiple holograms. Moreover, $\mathrm{CdF}_{2}$ offers also a much broader spectral range of scanning the hologram, as compared to AlGaAs. We may conclude then that semiconducting $\mathrm{CdF}_{2}$ crystals doped with bistable impurities can be considered as a possible efficient medium for writing thick and multiple holograms comparing favorably with other photorefractive materials [6].

\section{References}

[1] J.E. Dmochowski, J.M. Langer, Z. Kaliński, W. Jantsch, Phys. Rev. Lett. 56, 1735 (1986).

[2] J.E. Dmochowski, W. Jantsch, D. Dobosz, J.M. Langer, Acta Phys. Pol. A 73, 247 (1988).

[3] J.M. Langer, J. Phys. Soc. Jpn. Suppl. A 49, 207 (1980); Radiat. Effects 72, 55 (1983).

[4] A.I. Ryskin, A.S. Shcheulin, B. Koziarska, J.M. Langer, A. Suchocki, I.I. Buczinskaya, P.P. Fedorov, B.P. Sobolev, Appl. Phys. Lett. 67, 31 (1995).

[5] R.A. Linke, T. Thio. J.D. Chadi, G.E. Devlin, Appl. Phys. Lett. 65, 16 (1994).

[6] L. Hesselink, M.C. Bashaw, Opt. Quantum Electron. 25, S611 (1993). 